ISSN 1991- 8690

website: http:// jsci.utq.edu.iq
الترقيم الدولي • 1991 - 199

Email: utjsci@utq.edu.iq

\title{
Generalized GN'-Function for n-Variable
}

\author{
Hadeel A. Shubber \\ Abeer A.A. Aladhab \\ Department of Mathematics- College of Education \\ College of Art, \\ Thi-Qar University
}

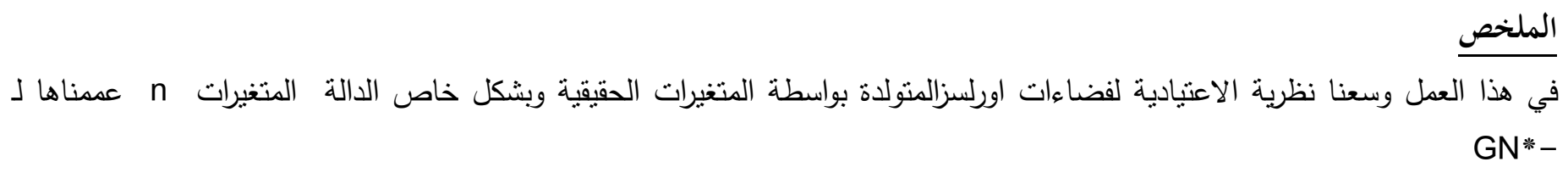

\section{ABSTRACT}

In this work, we extend the usual theory of Orlicz spaces generated by real valued $\mathrm{N}$-functions of a real variable. In particular, $\mathrm{GN}^{*}$-functions are the generalization of the variable $\mathrm{N}$-functions used by Portnov and the non-decreasing -function by Wang.

Firstly, we begin with new definitions:

\section{Introduction and Basic Concept}

In what follows $T$ will denote a space of point with $\sigma$-finite measure and $E^{n}$ a dimensional Euclidean space.

\section{Definition1.1[4]}

Orlicz space $\mathcal{L}_{\mathcal{M}}=\mathcal{L}_{\mathcal{M}}(\Omega, \mu)$ is a Banach space consisting of all $f \in S(\Omega, \mu)$ where $S(\Omega, \mu)$ is a ring of all measurable functions on the space with bounded measure $(\Omega, \mu)$. such that $\int_{\Omega} M(|f|) d \mu<\infty$,

With the Luxemburg Nakano norm $\|f\|_{M}=\inf \left\{\lambda>0: \int_{\Omega} M\left(\frac{|f|}{\lambda}\right) d \mu \leq 1\right\}$

Orlicz spaces $L m$ are natural generalization of $L p$ space, where $L p(I)$ consists of all the measurable functions $f$ defined on the interval I for which 


$$
\left(\int_{I}|f|^{p}\right)^{\frac{1}{p}}<\infty
$$

They have very rich topological and geometrical structures; they may possess peculiar properties that do not occur in an ordinary $L_{p}$ space.

\section{Definition1.2:[3]}

Let $M: I \rightarrow R$ be defined on some interval of the real line $\mathrm{R}$. A function $M$ is called convex if

$$
M\left(\frac{u_{1}+u_{2}}{2}\right) \leq \frac{1}{2}\left(M\left(u_{1}\right)+M\left(u_{2}\right)\right)
$$

$$
\text { for all } u_{1}, u_{2} \in I
$$

\section{Definition 1.3:[2]}

Let $M(t, x, y)$ be a real valued non-negative function defined on $T \times E^{n} \times E^{n}$ such that:

(i) $M(t, x, y)=0$ if and only if $x, y$ are the zero vectors $x, y \in E^{n}, \forall t \in T$

(ii) $M(t, x, y)$ is a continuous convex function of $x, y$ for each $t$ and a measurable function of $t$ for each $x, y$,

(iii) For each $t \in T, \lim _{\|x\|=\infty} \frac{M(t, x, y)}{\|x\| y \|}=\infty$, and

(iv)There are constants $d \geq 0$ and $d_{1} \geq 0$ such that

$$
\inf _{\substack{c \geq d \\ c \\ c^{\prime} \geq d_{1}}} k\left(t, c, c^{\prime}\right)>0
$$

Where

$$
\begin{gathered}
k\left(t, c, c^{\prime}\right)=\frac{M}{\bar{M}}\left(t, c, c^{\prime}\right) \\
\left.\bar{M}\left(t, c, c^{\prime}\right), c, c^{\prime}\right)=\sup _{\substack{|x| c c \\
y \mid=c^{\prime}}} M(t, x, y), \underline{M}\left(t, c, c^{\prime}\right)=\inf _{\mid \begin{array}{l}
|x| c c \\
y \mid=c^{\prime}
\end{array}} M(t, x, y) \quad \text { and if } d>0 \text { and } d_{1}>0,
\end{gathered}
$$

then $\bar{M}\left(t, d, d_{1}\right)$ is an integrable function of $t$. We call the function satisfying the properties (i)-(iv) a generalized $\mathrm{N} *$-function or a $\mathrm{GN}^{*}$-function. 


\section{Definition 1.4:}

Let $M\left(t, x_{1}, x_{2}, \ldots, x_{n}\right)$ be a real valued non-negative function defined on $T \times E^{n} \times E^{n} \times \underset{n-\text { times }}{\ldots} \times E^{n}$ such that: (i) $M\left(t, x_{1}, x_{2}, \ldots, x_{n}\right)=0$ if and only if $x_{1}, x_{2}, \ldots, x_{n}$ are the zero vectors $x_{1}, x_{2}, \ldots, x_{n} \in E^{n}, \forall t \in T$ (ii) $M\left(t, x_{1}, x_{2}, \ldots, x_{n}\right)$ is a continuous convex function of $x_{1}, x_{2}, \ldots, x_{n}$ for each $t$ and a measurable function of $t$ for each $x_{1}, x_{2}, \ldots, x_{n}$,

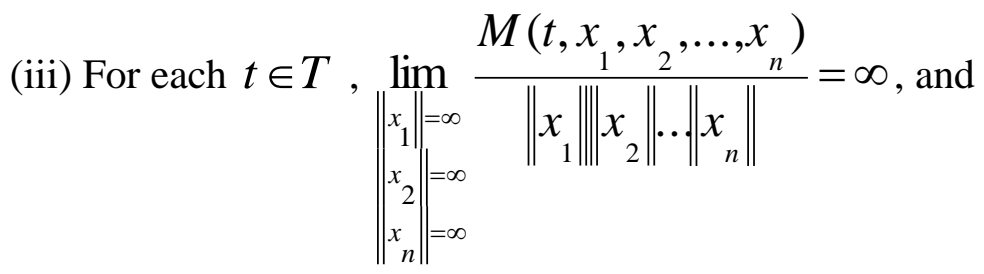

(iv)There are constants $d_{1} \geq 0, d_{2} \geq 0, \ldots, d_{n} \geq 0$ such that

$$
\inf _{t} \inf _{\substack{c \geq d \\ c^{1} \geq d \\ c^{2} \geq d^{2} \\ n}} k\left(t, c_{1}, c_{2}, \ldots, c_{n}\right)>0
$$

Where

$k\left(t, c_{1}, c_{2}, \ldots, c_{n}\right)=\frac{\underline{M}\left(t, c_{1}, c_{2}, \ldots, c_{n}\right)}{\bar{M}\left(t, c_{1}, c_{2}, \ldots, c_{n}\right)}$,

$\bar{M}\left(t, x_{1}, c_{2}, \ldots, c_{n}\right)=\sup _{\mid \begin{array}{l}x_{1} \mid=c_{1} \\ x_{2} \mid=c_{2} \\ x_{n} \mid=c_{n}\end{array}} M\left(t, x_{1}, x_{2}, \ldots, x_{n}\right), \quad M\left(t, c_{1}, c_{2}, \ldots, c_{n}\right)=\inf _{\mid \begin{array}{l}x_{1} \mid=c_{1} \\ x_{2} \mid=c_{2} \\ x_{n} \mid=c_{n}\end{array}} M\left(t, x_{1}, x_{2}, \ldots, x_{n}\right)$

and if $d_{1}>0, d_{2}>0, \ldots, d_{n}>0$, then $\bar{M}\left(t, d_{1}, d_{2}, \ldots, d_{n}\right)$ is an integrable function of $t$. We call the function satisfying the properties (i)-(iv) a generalized N'-function or a GN'-function.

\section{Example:}

GN'-functions are coordinate independent and are not necessarily symmetric. Therefore, such functions as $M\left(t, x_{1}, x_{2}, \ldots, x_{n}\right)$

$M\left(t, x_{1}, x_{2}, \ldots, x_{n}\right)=\left(x_{1}+y_{1}\right)^{2}+\left(x_{2}+y_{2}\right)^{2}$

$$
+\left[\left(x_{1}+y_{1}\right)-\left(x_{2}+y_{2}\right)-\ldots-\left(x_{n}+y_{n}\right)\right]^{2}
$$


which are nt non-decreasing (as defined in [3]) are allowed in the class of GN'-functions. The next theorem illustrates this point.

\section{Theorem 1.5:[2]}

If $M(t, x, y)$ is a $\mathrm{GN}^{*}$-function and $A$ is an orthogonal linear transformation defined on $E^{n} \times E^{n}$, with the range in $E^{n} \times E^{n}$, then $\tilde{M}(t, x, y)=M(t, A x, A y)$ is a $\mathrm{GN}^{*}$-function.

\section{Theorem 1.6:[2]}

A necessary and sufficient condition that (1.3.1) holds is that if $|x| \leq|z|$ and $|y| \leq|w|$, then there exists constants $K \geq 1, d \geq 0 \quad$ and $\quad d^{\prime} \geq 0 \quad$ such that $M(t, x, y) \leq K M(t, z, w)$ for each $t$ in $T,|x| \geq d$ and $|y| \geq d^{\prime}$.

\section{Remark:[2]}

It is interesting to note that if $M(t, x, y)$ is a $\mathrm{GN}^{*}$-function, then $2 \hat{M}(t, x, y)=M(t, x, y)+\tilde{M}(t, x, y)$ is also a $\mathrm{GN}^{*}$-function where $\tilde{M}(t, x, y)$ is defined as in Theorem 1.5. This means we can construct a symmetric (in $x$ and $y$ ) $\mathrm{GN}^{*}$-function from one which does not possess this property.

For, if $\tilde{M}(t, x, y)=M(t,-x,-y)$, then $\hat{M}(t, x, y)$ is clearly symmetric in $x$ and $y$.

Property (iv) of the definition 1.3 provides the condition which allows a natural gen eralization from $\mathrm{GN}^{*}$-function of a real variable to those of several real variables. Let us observe that the function $\bar{M}\left(t, c, c^{\prime}\right)$ is also a $\mathrm{GN}^{*}$-function of a real nonnegative variable $c$ and $c^{\prime}$. On the other hand, $M\left(t, c, c^{\prime}\right)$ need not even be convex in $c$ and $c^{\prime}$.

Since $\underline{M}\left(t, c, c^{\prime}\right) \leq M(t, x, y) \leq \bar{M}\left(t, c, c^{\prime}\right)$ for each $x$ and $y$ such that $|x|=c$ and $|y|=c^{\prime}$ we would like to find a $\mathrm{GN}^{*}$-function which bounds

$\underline{M}\left(t, c, c^{\prime}\right)$ from below for all $c$ and $c^{\prime}$. If $d=0$ and $d^{\prime}=0$ in Theorem (1.6), then $K^{-1} \bar{M}\left(t, c, c^{\prime}\right)$ would do.

One might accomplish the construction of such a function by taking the supremum of a class of convex functions bounding $\underline{M}\left(t, c, c^{\prime}\right)$ from below. This function would be convex. However, this class may be empty. The next theorem shows that this is not the case whenever $M(t, x, y)$ is a GN*-function. The construction employed can be applied to more general settings than those which exist here. 


\section{Theorem 1.7:[2]}

If $M(t, x, y)$ is a $\mathrm{GN}^{*}$-function and $\underline{M}\left(t, c, c^{\prime}\right)$ is defined as above, then there exists a $\mathrm{GN}^{*}$-function $R\left(t, c, c^{\prime}\right)$ such that $R\left(t, c, c^{\prime}\right) \leq \underline{M}\left(t, c, c^{\prime}\right)$ for all $c \geq 0$ and $c^{\prime} \geq 0$.

\section{Generalized GN'-Function}

\section{Theorem 2.1:}

If $M\left(t, x_{1}, x_{2}, \ldots, x_{n}\right)$ is a $\mathrm{GN}^{\prime}$-function and $A$ is an orthogonal linear transformation defined on $E^{n} \times E^{n} \times \ldots \times E^{n}$, with the range $\quad$ in $E^{n} \times E^{n} \times \ldots \times E^{n}$, then $\tilde{M}\left(t, x_{1}, x_{2}, \ldots, x_{n}\right)=M\left(t, A x_{1}, A x_{2}, \ldots, A x_{n}\right)$ is a GN'-function.

\section{Proof:}

Properties (i)-(iv) when applied to $\tilde{M}\left(t, x_{1}, x_{2}, \ldots, x_{n}\right)$ follow immediately from the same properties for $M\left(t, x_{1}, x_{2}, \ldots, x_{n}\right)($ see $[5$, Th 8.1$])$.

The next theorem characterizes a part of the property (iv) in the definition 1.4 and provides a means of comparing function values at different points for GN'-function when $\left|x_{1}\right|,\left|x_{2}\right|, \ldots,\left|x_{n}\right|$ are large.

\section{Theorem 2.2:}

A necessary and sufficient condition that (1.4.1) holds is that if $\left|x_{1}\right| \leq\left|y_{1}\right|,\left|x_{2}\right| \leq\left|y_{2}\right|, \ldots,\left|x_{n}\right| \leq\left|y_{n}\right|$ then there exists constants

$$
K \geq 1, d_{1} \geq 0, d_{2} \geq 0, \ldots, d_{n} \geq 0
$$
such that $M\left(t, x_{1}, x_{2}, \ldots, x_{n}\right) \leq K M\left(t, y_{1}, y_{2}, \ldots, y_{n}\right)$ for each $t$ in $T,\left|x_{1}\right| \geq d_{1},\left|x_{1}\right| \geq d_{1}, \ldots,\left|x_{n}\right| \geq d_{n}$

\section{Proof:}

If (1.4.1) is true, then there exists constants $d_{1} \geq 0, d_{2} \geq 0, \ldots, d_{n} \geq 0$ such that $\tau(t)=\inf _{\substack{c \\ 1 \\ 1}} k\left(t, c_{1}, c_{2}, \ldots, c_{n}\right)>0$ for each $t$ in $T$. By the definition of $K\left(t, c_{1}, c_{2}, \ldots, c_{n}\right)$ this means 


$$
M\left(t, x_{1}, x_{2}, \ldots, x_{n}\right) \geq \underline{M}\left(t,\left|x_{1}\right|,\left|x_{2}\right|, \ldots,\left|x_{n}\right|\right) \geq \tau(t) \bar{M}\left(t,\left|x_{1}\right|,\left|x_{2}\right|, \ldots,\left|x_{n}\right|\right)(
$$

for any $x_{1}, x_{2}, . ., x_{n}$ such that $\left|x_{1}\right|=c_{1} \geq d_{1},\left|x_{2}\right|=c_{2} \geq d_{2}, \ldots,\left|x_{n}\right|=c_{n} \geq d_{n}$.

On the other hand, if $d_{1} \leq\left|x_{1}\right| \leq\left|y_{1}\right|, d_{2} \leq\left|x_{2}\right| \leq\left|y_{2}\right|, \ldots, d_{n} \leq\left|x_{n}\right| \leq\left|y_{n}\right| \quad$ then the convexity of $M\left(t, x_{1}, x_{2}, . ., x_{n}\right)$ and $M(t, 0,0, \ldots, 0)=0$ yields

$$
\bar{M}\left(t,\left|y_{1}\right|,\left|y_{2}\right|, \ldots,\left|y_{n}\right|\right) \geq \sup _{\mid \begin{array}{l}
z_{1}||\left|x_{1}\right| \\
z_{2}|=| x_{2} \\
z_{n}|=| x_{n} \mid
\end{array}} M\left(t, z_{1}, z_{2}, \ldots, z_{n}\right)
$$

By combining (2.2.1) and (2.2.2), we arrive at

$$
M\left(t, y_{1}, y_{2}, \ldots, y_{n}\right) \geq \tau(t) \sup _{\substack{z_{1}|=| x_{1}\left| \\z_{2}=\right| x_{2} \\ z_{n}|=| x_{n}}} M\left(t, z_{1}, z_{2}, \ldots, z_{n}\right) \geq K^{-1} M\left(t, x_{1}, x_{2}, \ldots, x_{n}\right),
$$

When ever $d_{1} \leq\left|x_{1}\right| \leq\left|y_{1}\right|, d_{2} \leq\left|x_{2}\right| \leq\left|y_{2}\right|, \ldots, d_{n} \leq\left|x_{n}\right| \leq\left|y_{n}\right|$ where $\left.K^{-1}=\inf \tau(t)\right\rangle 0$.

The converse follows easily from the condition in the theorem.

\section{Remark:}

It is interesting to note that if $M\left(t, x_{1}, x_{2}, \ldots, x_{n}\right)$ is a $\mathrm{GN}^{\prime}$-function, then $2 \hat{M}\left(t, x_{1}, x_{2}, \ldots, x_{n}\right)=M\left(t, x_{1}, x_{2}, \ldots, x_{n}\right)+\tilde{M}\left(t, x_{1}, x_{2}, \ldots, x_{n}\right) \quad$ is also a GN'-function where $\tilde{M}\left(t, x_{1}, x_{2}, \ldots, x_{n}\right)$ is defined as in Theorem 2.1. This means we can construct a symmetric (in $x_{1}, x_{2}, . ., x_{n}$ ) GN'-function from one which does not possess this property.

For, if $\tilde{M}\left(t, x_{1}, x_{2}, \ldots, x_{n}\right)=M\left(t,-x_{1},-x_{2}, \ldots,-x_{n}\right)$, then $\hat{M}\left(t, x_{1}, x_{2}, \ldots, x_{n}\right)$ is clearly symmetric in $x_{1}, x_{2}, . ., x_{n}$.

Property (iv) of the definition 1.4 provides the condition which allows a naturral generalization from N'function of a real variable to those of several real variables. Let us observe that the function $\bar{M}\left(t, c_{1}, c_{2}, \ldots, c_{n}\right)$ is also a GN'-function of a real nonnegative variable $c_{1}, c_{2}, \ldots, c_{n}$.On the other hand, $M\left(t, c_{1}, c_{2}, \ldots, c_{n}\right)$ need not even be convex in $c_{1}, c_{2}, \ldots, c_{n}$. 
Since $\underline{M}\left(t, c_{1}, c_{2}, \ldots, c_{n}\right) \leq M\left(t, x_{1}, x_{2}, \ldots, x_{n}\right) \leq \bar{M}\left(t, c_{1}, c_{2}, \ldots, c_{n}\right)$ for each $c_{1}, c_{2}, \ldots, c_{n}$ such that $\left|x_{1}\right|=c_{1},\left|x_{2}\right|=c_{2}, \ldots,\left|x_{n}\right|=c_{n}$ we would like to find a GN'-function which bounds

$\underline{M}\left(t, c_{1}, c_{2}, \ldots, c_{n}\right)$ from below for all $c_{1}, c_{2}, \ldots, c_{n}$. If $d_{1}=0, d_{2}=0, \ldots, d_{n}=0$ in Theorem (2.2), then $K^{-1} \bar{M}\left(t, c_{1}, c_{2}, \ldots, c_{n}\right)$ would do.

One might accomplish the construction of such a function by taking the supremum of a class of convex functions bounding $\underline{M}\left(t, c_{1}, c_{2}, \ldots, c_{n}\right)$ from below. This function would be convex. However, this class may be empty. The next theorem shows that this is not the case whenever $M\left(t, x_{1}, x_{2}, \ldots, x_{n}\right)$ is a GN'function. The construction employed can be applied to more general settings than those which exist here.

\section{Theorem 2.3:}

If $M\left(t, x_{1}, x_{2}, \ldots, x_{n}\right)$ is a GN'-function and $\underline{M}\left(t, c_{1}, c_{2}, \ldots, c_{n}\right)$ is defined as above, then there exists a GN'-function $R\left(t, c_{1}, c_{2}, \ldots, c_{n}\right)$ such that $R\left(t, c_{1}, c_{2}, \ldots, c_{n}\right) \leq \underline{M}\left(t, c_{1}, c_{2}, \ldots, c_{n}\right)$ for all $c_{1} \geq 0, c_{2} \geq 0$ $, \ldots, c_{n} \geq 0$

\section{Proof:}

Since $\underline{M}\left(t, c_{1}, c_{2}, \ldots, c_{n}\right)$ satisfies property (iii) of the definition 1.4 , given any $d>0$ there are $c_{1}^{\prime}>0, c_{2}^{\prime}>0, \ldots, c_{n}^{\prime}>0$ such that $\underline{M}\left(t, c_{1}, c_{2}, \ldots, c_{n}\right) \geq d c_{1} c_{2} \ldots c_{n}$ whenever $c_{1} \geq c_{1}^{\prime}, c_{2} \geq c_{2}^{\prime}, \ldots, c_{n} \geq c_{n}^{\prime}$. Let us define the function

$$
P\left(t, c_{1}, c_{2}, \ldots, c_{n}\right)=\left\{\begin{array}{l}
\sup _{n} \frac{M\left(t, c_{1} w_{1}, c_{2} w_{2}, \ldots, c_{n} w_{n}\right)}{w_{1} w_{2} \ldots w_{n}} \quad \text { if } \quad c_{1} \geq c_{1}^{\prime}, c_{2} \geq c_{2}^{\prime}, \ldots, c_{n} \geq c_{n}^{\prime} \\
0<w_{1} \leq 1 \\
0<w_{2} \leq 1 \\
0<w_{n} \leq 1 \\
c_{n} w_{1} \geq c_{1}^{\prime} \\
c_{2} w_{2} \geq c_{2}^{\prime} \\
c_{2} w_{n} \geq c^{\prime} \\
M_{n}\left(t, c_{1}, c_{2}, \ldots, c_{n}\right) \quad \text { if } \quad 0 \leq c_{1}<c_{1}^{\prime}, 0 \leq c_{2}<c_{2}^{\prime}, 0 \leq c_{n}<c_{n}^{\prime}
\end{array}\right.
$$


Then, it is easy to show that (i) $P\left(t, a c_{1}, a c_{2}, \ldots, a c_{n}\right) \leq a^{n} P\left(t, c_{1}, c_{2}, \ldots, c_{n}\right)$ for $0 \leq a \leq 1$,

(ii) $\left\{\frac{p\left(t, c_{1}, c_{2}, \ldots, c_{n}\right)}{c_{1} c_{2} . . c_{n}}\right\}$ is a non-decreasing function of $c_{1}, c_{2}, \ldots$ and $c_{n}$, and (iii) $P\left(t, c_{1}, c_{2}, \ldots, c_{n}\right)$ is finite for each $c_{1}, c_{2}, .$. and $c_{n}$. We now obtain the desired function $R\left(t, c_{1}, c_{2}, \ldots, c_{n}\right)$ by defining

$$
R\left(t, c_{1}, c_{2}, \ldots, c_{n}\right)=\int_{0}^{c} \int_{0}^{c} \ldots \int_{0}^{c} Q\left(t, s_{1}, s_{2}, \ldots, s_{n}\right) d s_{1} d s_{2} \ldots d s_{n}
$$

where

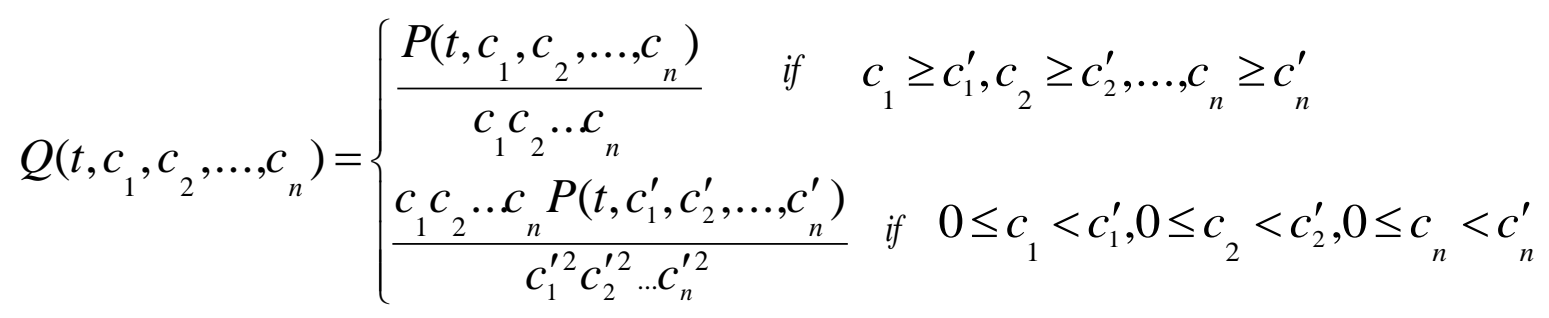

Immediately we have

$$
R\left(t, c_{1}, c_{2}, \ldots, c_{n}\right) \leq c_{1} c_{2} . . c_{n} Q\left(t, c_{1}, c_{2}, \ldots, c_{n}\right)=P\left(t, c_{1}, c_{2}, \ldots, c_{n}\right) \leq \underline{M}\left(t, c_{1}, c_{2}, \ldots, c_{n}\right) .
$$

It is not difficult to show that $R\left(t, c_{1}, c_{2}, \ldots, c_{n}\right)$ is also a GN'-function.

\section{References}

1.N.Corothers (2000):"A short course on Banach space theory ".Bowling Green state university.

2.H.A.Hassen (2007):"Vector Valued Orlicz Space Generalized GN*-function"Msc.thesis.Kufa University. Department of Mathematic.College of Education.

3.A.Roberts and D.Varberg (1973):"Convex functions ".Newyork.

4.W.Orlicz (1932):"Vber eine gewisse klasic von Räumen vom Typus".BBull.Int.Acad Polon.Sci.207220.

5. Wang Sheng-Wang (1963):"Convex function of several variables and vector valued Orlicz spaces".Bull.Aead.POLOn.Sc.ser.math. Astr.et Phys.11, 279-284. 